\title{
A study on the bridge of music intelligence to Chinese comprehension -- Hope and limitation?
}

\author{
Wei-Yu Liu \\ National Dong Hwa University \\ Taiwan, Province of China
}

\begin{abstract}
This study aims to use the theory of multiple intelligences to explore the strategies of music intelligence bridging Chinese comprehension and their impacts on students' learning. An action research was conducted since the purposes of this study are both to test multiple intelligences theory and to improve Chinese teaching. Several models and approaches have been developed to apply multiple intelligences theory into practice in K-8 classrooms. Baum et al (2005) identified five pathways to apply multiple intelligences theory in the elementary classroom; they are Explorations, Building on Strengths, Understanding, Authentic Problems, and Talent Development. While Viens and Kallenbach (2004) labeled the first fourth as MI Reflections, Bridging Students' Areas of Strengths to Areas of Challenge, Entry/Exit Points, and Projects. The approach of Bridging Students' Areas of Strengths to Areas of Challenge is to create a "bridge" from students' MI strengths to appropriate learning strategies, emphasizing using students' particular strengths to assist in areas of particular difficulty (Viens \& Kallenbach, 2004). The models of Vocabulary-in-Song bridging Chinese reading comprehension was built and discussed. Teaching skills which must be coupled with the bridging model were discussed. The hope and limitation of Vocabulary-in-Song bridging Chinese reading comprehension were showed.
\end{abstract}

\section{THE PURPOSE AND IMPORTANCE OF THE STUDY}

According to the current official classification, there are twelve indigenous groups in Taiwan, the Amis, Atayal, Paiwan, Bunun, Rukai, Puyuma, Saisiat, Tsou, Thao, Yamis, Karalan and the Truku. The total population of these twelve indigenous groups was 458,326 in 2005. The Truku, with a population of 9,262 is the seventh largest of all of Taiwan's indigenous groups. The indigenous groups in Taiwan belong to the Austronesian language family. However, the national language spoken in Taiwan, Chinese, belongs to the Han language family. Learning to read and write the mainstream language is difficult for many indigenous children in Taiwan, just like other aboriginal pupils in the world.

Indigenous people have long been bothered by their low academic performance in the school. Using the assimilation policy to enhance their academic performance has been proved to have little effect. People who believe in cultural-difference or multicultural education theories argue that it is the lack of cultural-adaptive content and pedagogy that indigenous people lose skills, attitudes, and knowledge needed to function well (LeCompte, 1987; Wilson, 1991).

Gardner, arguing that our culture had defined intelligence too narrowly, proposed the existence of at least eight basic intelligences, that is, verbal/linguistic intelligence, logicalmathematical intelligence, bodily-kinesthetic intelligence, musical/rhythmic intelligence, visual/spatial intelligence, intrapersonal intelligence, interpersonal intelligence, and naturalist intelligence. We all have different combinations of intelligences (Gardner, 1983; 1999). 
There have been a lot of researches dealt with the issue of cultural-adaptive contents (Gay, 2000), but little research dealt with the issue of intelligence-adaptive pedagogy. Multiple intelligences theory makes its greatest contribution to education by suggesting that teachers need to expand their repertoire of techniques, tools, and strategies beyond the typical linguistic and logical ones predominantly used in the classroom (Armstrong, 2000). The hypothesis of this study is lacking of emphasis on teaching aboriginal pupils through their developed intelligences results in their low academic performance.

This study aims to use the theory of multiple intelligences to explore the strategies of music intelligence bridging Chinese comprehension and their impacts on students' learning. The model of Vocabulary-in-Song bridging comprehension is built and discussed. Teaching skills which must be coupled with the bridging model were discussed. The hope and limitation of Vocabulary-in-Song bridging Chinese reading comprehension were showed.

\section{PERSPECTIVE}

Howard Gardner refined intelligence as "a bio-psychological potential to process information that can be activated in a cultural setting to solve problems or create products that are of value in a culture". He laid out eight criteria and identified eight intelligences that satisfactorily fulfilled these criteria. The eight intelligences include verbal/linguistic, logical-mathematics, bodily-kinesthetic, musical/rhythmic, visual/spatial, intrapersonal, interpersonal, and naturalist intelligences. Each intelligences is different not only neurologically, but in the symbol systems they belong to, the tools they call on, the core or sub-abilities included in each, and how each is utilized in the real world (Gardner, 1999).

Multiple intelligences theory makes its greatest contribution to education by suggesting that teachers need to expand their repertoire of techniques, tools, and strategies beyond the typical linguistic and logical ones predominantly used in the classroom (Armstrong, 2000). Using multiple intelligences informed approaches usually involves an iterative process between curriculum/instruction and assessment. Multiple intelligences theory helps teachers frame activities so that many entry points into the subject matter are available to students. Teachers use the knowledge they have accumulated about students' intelligences and preferences to inform subsequent instruction. Teachers who offer different pedagogical approaches and allow exploration of differing perspectives create the possibility of reaching more students more effectively (Gardner, 1999).

Baum, Viens and Slatin (2005) have identified four pathways teachers take to apply multiple intelligences theory in their practice. They are multiple intelligences reflections, bridging, entry/exit points, and projects. The multiple intelligences reflection pathway uses multiple intelligences as a basis to reflect on and identify students' strengths and preferences. The bridging pathway emphasizes using students' particular strengths to assist in areas of particular difficulty. The entry/exist points pathway provides a range of multiple intelligences informed "entry points" into a topic and "exit points" for students to demonstrate their learning. The projects pathway uses multiple intelligences theory as a framework to develop project-based curriculum.

The bridging pathway uses multiple intelligences to help students master the basic skills of literacy and math. The approach of Bridging Students' Areas of Strengths to Areas of Challenge is to create a "bridge" from students' MI strengths to appropriate learning strategies, emphasizing using students' particular strengths to assist in areas of particular difficulty. This pathway is based on two assumptions. First, students achieve better when allowed to pursue learning from a position of strength. (Baum, Viens and Slatin, 2005). 
The second assumption is the acknowledgement that using a multiple intelligences approach can enhance the learning process. Multiple intelligences theory can help to explain individual differences in how students learn. Different intelligence symbol system attracts certain people and influences both memory and attention. Using a multiple intelligences frame to understand individual differences in how students learn can assist in explaining why some students are successful and others are not. These observations can contribute to instructional strategies that tap individual students' intellectual strengths and allow them to be successful in developing literacy (Baum, Viens \& Slatin, 2005).

Music intelligence is the capacity to think in music, to be able to hear patterns, recognize them, remember them, and perhaps manipulate them. Its key abilities involve perceiving and understanding patterns of sound, creating and communicating meaning from sound (Gardner, 1999; Viens \& Kallenbach, 2004). Reading comprehension is assisted by using music to help students identify context clues, build story maps and develop their own thoughts and feelings about a story (Kolb, 1996).

Mora (2000) suggested that all the intelligences of our learners should be addressed and developed in the classroom, including music intelligence, which is highly relevant for language teaching. From an ontological point of view, sounds are the roots of both music and speech. Music and language share several features. On the one hand, both stem from the processing of sounds; on the other, both are used by their authors/speakers to convey a message (Mora, 2000). About integration, Wiggins (2001) developed five levels of integrated curricula for music teacher or artists: teaching-tool connections, topic connections, thematic or content connections, conceptual connections, and process connections. MI theory didn't point out which kind bridging curriculum is the best model, so teacher must design bridging curriculum according to his students and the teaching environment.

Harp (1988) declares that music and language arts are complementary activities. Research evidence shows that there is a connection between music and language arts in the early primary grades. There are two types of research that demonstrate the relationship between music and language arts: the first is that music can be used as a tool to teach language arts or other content areas and the second is that the study of music as its own discipline transfers to other content areas (Benson, 2000).

This study aims to explore topic or content connections Vocabulary-in-Song bridging Chinese comprehension. It is crucial for pupils who are linguistic intelligence under developed but musical intelligence well developed.

\section{Description of pupils}

\section{METHODS}

The action research was conducted in a third grade classroom with 17 Truku pupils, 11 boys, 6 girls. Most of them cannot concentrate their attention more than 10 minutes in Chinese class. Their average Chinese comprehension performance is in the second grade level. Music intelligence was most students' developed intelligence.

\section{Description of collaborated action researchers}

Misa and the author were collaborated action researchers in this study. Misa was the third grade class teacher, who taught Chinese and Music. She liked the theory of multiple intelligences and appreciated to have the opportunity to try applying MI to Child's Chinese learning. She thought her class need help to improve their Chinese reading comprehension. 
Misa was the teaching actor in this study. The author of this study took the roles of organizer, challenger, resource person, change agent, reflective researcher and friend.

\section{Action Research Lesson Design}

The research team operated in a highly collaborative manner, engaging in the cyclic process of planning action, monitoring the action, implementing the action, collecting and analyzing data, reviewing and evaluating data, and reflecting on its actions.

The theory of multiple intelligences served as a basis for designing the lesson. The researcher uses MI to develop activities and learning strategies that are tailored to students' strengths. That is, the researcher's goal was to apply our understanding of MI theory, and of the targeted students' particular strengths and preferences, to develop different ways to engage students in Chinese reading comprehension.

By analyzing the nature of Chinese texts and music intelligence, the researcher employs Vocabulary-in-Song to design the lesson plan. Vocabulary-in-Song uses melody element, one of the core elements of music intelligence, to design. From an ontological point of view, sounds are the roots of both music and speech. Music and language share several features. On the one hand, both stem from the processing of sounds; on the other, both are used by their authors/speakers to convey a message (Mora, 2000).

The researcher uses vocabulary to compose songs which melody is well known by students. Students will learn to sing the song first, and then read Chinese text and vocabulary. While teacher teaches vocabulary, she will first read it with rhyme and pitch, followed by students. Then she let students recognize the same vocabulary shown on Vocabulary-in-Song. Third, she explains the meaning of vocabulary with multiple ways, including the context of Vocabulary-inSong. Fourth, she helps students make sentences with the vocabulary. If students can not make any sentences by themselves, they can use Vocabulary-in-Song to make sentences. Fifth, students use their own words to make clear of the meaning of Vocabulary-in-Song lyrics.

At the end of each three units, students take Vocabulary-in-Song comprehension tests and sentence-making worksheets.

\section{Data Collection and Analysis}

Several data collection techniques were used to ensure triangulation of the data. In the planning action cycle, lesson plans were collected and analyzed. In the monitoring and implementing action cycle, all Chinese teaching class were videotaped; the class teaching videotapes were collected and transcribed. Field-notes were taken, collected and transcribed. The reflective journals, student learning worksheets, sentence-making worksheets and Vocabulary-in-Song comprehension tests were collected and analyzed. The Chinese learning attitude questionnaire was conducted, collected and analyzed.

Over the duration of the study, the action research group held 3 formal meeting; all meetings were audio-taped, transcribed and analyzed. Semi-structured interviews were conducted with each participant at the beginning and end of the action research project. Informal conversational interview occurred after action research meetings, after classroom teaching sessions, and after regular school hours. Interviews and recreated notes were transcribed, collected and analyzed. 


\section{RESULTS AND DISCUSSION}

Vocabulary-in-Song (Music intelligence) bridges Chinese comprehension teaching

1. The teaching model of Vocabulary-in-Song bridging Chinese comprehension is as following: First, students sing the song. Second, students recognize vocabulary shown on Vocabulary-in-Song. Third, teacher explains the meaning of vocabulary with multiple ways, including the context of Vocabulary-in-Song. Fourth, students make sentences with the vocabulary. Fifth, students use their own words to make clear of the meaning of Vocabulary-in-Song lyrics.

2. A requirement for Vocabulary-in-Song model works is that students will have enough time to master Vocabulary-in-Song.

3. The key aspect of Vocabulary-in-Song bridging Chinese comprehension model lays in pupils master the meaning of lyrics. Therefore, teacher successfully explains the meaning of vocabulary with multiple ways, including the context of Vocabulary-in-Song is crucial.

4. Teaching step fifth, students use their own words to make clear of the meaning of Vocabulary-in-Song lyrics, could be put either after students making sentences or text content explanation.

5. The choice of Vocabulary-in-Song melody is very important. Melody which familiar to students, slower, and harmony with content is the best one.

6. The Vocabulary-in-Song bridging Chinese comprehension model is not good for Vocabulary with abstract meaning. To interpret vocabulary with abstract meaning like treasure time, experienced activities, examples and discussion work better.

7. Teachers need both literature and music capacity to employ Vocabulary-in-Song bridging Chinese comprehension model.

The effect of Vocabulary-in-Song method to students' Chinese comprehension

Vocabulary-in-Song comprehension tests, sentence-making worksheets, and Chinese learning attitude questionnaire were analyzed to explore the effect of Vocabulary-in-Song method to students' Chinese comprehension. The results are as following:

1. For the Vocabulary-in-Song comprehension tests, there are eight knowledge level test questions in this test. Pupils' average correct ratio is 68.3\%. There are eleven comprehension level test questions in this test. Pupils' average correct ratio is $69.7 \%$. There are forty application level test questions in this test. Pupils' average correct ratio is $72.2 \%$.

2. For the sentence-making worksheets, there are 36 vocabulary and phrases for pupils to make sentences. Pupils' average is three out of four point rating system. That is, students make sentences with integrity but not smooth and clear. For example, "I should sleep at home, why I run to house of others?"

3. For Chinese learning attitude questionnaire, $75 \%$ like learning Chinese very much; $25 \%$ no comment.

4. The reasons why they like Chinese? 25\% like Chinese class because Chinese is used daily. $37.5 \%$ pupils like Chinese class because they learned a lot in class. $12.5 \%$ pupils like Chinese class because it is interesting. $25 \%$ no comment.

5. The reasons why they don't like Chinese class? 25\% don't like Chinese class because it was boring; $75 \%$ no comment.

In summary, this paper uses scientific method to test the idea of bridging pathway of multiple intelligences, the intelligence-adaptive pedagogy. This study establishes Vocabulary-in-Song bridging Chinese comprehension teaching model and related teaching skills. The hope of Vocabulary-in-Song model is it can help Chinese low achievement pupils to have above average performance. The limitations of Vocabulary-in-Song model is it is time-consumed, not good for 
Vocabulary with abstract meaning, and teachers need to have both literature and music capacity. More details will be discussed in the following full paper.

\section{References}

Armstrong, T. (2000) . Multiple intelligences in the classroom. (2nd ed.). Alexandria, VA: Association for Supervision and Curriculum Development.

Baum, S., Viens J. \& Slatin, B. (2005). Multiple intelligences in the elementary classroom: pathways to thoughtful practice. New York: Teachers College Press.

Benson L. F. (2000). Using music to develop language arts skills in the classroom: a guide for the classroom teacher. The Graduate School of Education and Psychology at Pepperdine University. Unpublished dissertation.

Gardner, H. (1983) . Frames of mind: The theory of multiple intelligences. New York: Basic Books.

Gardner, H. (1999) . Intelligence reframed: Multiple intelligences for the 21 ${ }^{\text {st }}$ century. New York: Basic Books.

Gay, G. (2000). Culturally Responsive Teaching: theory, research, and practice. New York: Teachers College Press.

Harp, B. (1988). Why are your kids singing during reading time? The Reading Teacher, January, 454-456.

Kolb, G.. R. (1996). Read with a beat: developing literacy through music and song. The Reading Teacher, 50(1), 76 77.

LeCompte, N. D. (1987). The cultural context of dropping out: Why good dropout programs don't work. Paper presented at the American Association for the Advancement of Sciences, Chicago.

Mora, C., F. (2000). Foreign language acquisition and melody singing. ELT Journal, 54. (2.), p146-152

Viens, J. \& Kallenbach, S. (2004). Multiple intelligences and Adult Literacy: a sourcebook for practitioners. NY: Teachers College.

Wiggins, R. A. (2001). Interdisciplinary curriculum: music educator concerns. Music Educators Journal, 87. (5.), 4045 .

Wilson, P. (1991). Trauma of Sioux Indian high school students. Anthropology and Education Quarterly, 22, 367383. 